\title{
Functional Outcome Evaluation of Septorhinoplasty for Nasal Obstruction
}

\author{
Farah Dayana Zahedi ${ }^{1} \cdot$ Salina Husain ${ }^{1} \cdot$ Balwant Singh Gendeh $^{1}$
}

Received: 12 June 2015/ Accepted: 8 September 2015/Published online: 11 September 2015

(C) Association of Otolaryngologists of India 2015

\begin{abstract}
A prospective single blinded interventional study was held in Otorhinolaryngology Clinic, Universiti Kebangsaan Malaysia Medical Centre in August 2010 until November 2012 to evaluate the functional outcome of septorhinoplasty objectively and subjectively. Objective assessment was done using rhinomanometry and subjective assessment using Nasal Obstruction Symptoms Evaluation (NOSE) scale and Health-Related Quality of Life Questionnaires (HRQOL) in Rhino Surgery. All measurements were taken preoperatively and 3 months postoperatively. A total of 29 patients were enrolled and completed the study. Septorhinoplasty was commonly performed in Malays and Indians and rare amongst Chinese, with age ranged from 18 to 54 years. Majority had no history of trauma. Twisted nose was the most common external nose abnormality followed by crooked and saddle nose. All patients had internal valve insufficiency. There were significant improvement of the total and of all the parameters in the NOSE scale post septorhinoplasty $(p<0.05)$. Furthermore, there were significant improvement in total and in all parameters in HRQOL score post septorhinoplasty $(p<0.05)$. There were improvements in the rhinomanometry data bilaterally during inspiration and expiration, but were not statistically significant $(p>0.05)$. Significant correlations were noted in the improvement between the two subjective assessments (NOSE scale and HRQOL score). However, there was no significant
\end{abstract}

Farah Dayana Zahedi

anna_firra82@yahoo.com.au

1 Department of Otorhinolaryngology-Head and Neck Surgery, Universiti Kebangsaan Malaysia Medical Centre, Jalan Ya'acob Latif, Bandar Tun Razak, 56000 Cheras, Kuala Lumpur, Malaysia correlation in the improvement between the subjective (NOSE scale and HRQOL score) with objective (rhinomanometry score) assessments. Septorhinoplasty improves the nasal airflow and quality of life of patients with nasal obstruction.

Keywords Nasal obstruction - Rhinoplasty · Quality of life · Rhinomanometry

\section{Introduction}

Nasal obstruction is one of the most common presenting complaints in otorhinolaryngology practice [1]. Nasal obstruction causes are divided into fixed components that may need surgical repair, and dynamic components which is treatable medically. The most common fixed anatomic components causing nasal airway obstruction is deviated nasal septum [2], and nasal valve compromise as the primary cause for nasal airway obstruction has been well documented [3]. Septorhinoplasty addresses the fixed component of nasal obstruction due to anatomical abnormalities that failed medical treatment due to nasal valve insufficiency. Rhinomanometry performed pre and postoperatively on 160 patients revealed that septoplasty alone resulted in modest improvement in mean nasal airflow, whereas external or internal valvular reconstruction resulted in more marked improvement in nasal airflow. Significant improvements was noted in patients who underwent septorhinoplasty (combination of septoplasty with internal and external valvular reconstruction) $[2,4]$.

Nasal obstruction is associated with a significant decrease in disease specific quality of life [5]. However, QOL measurements after treatment are very subjective. Numerous studies have tended to objectify the severity of 
obstruction using rhinomanometry, acoustic rhinomanometry and nasal peak flow [6]. Majority studies evaluate the effect of septoplasty and rhinoplasty alone and measure the objective and subjective of septoplasty or rhinoplasty separately. In this study - the objective assessment using rhinomanometry is combined with subjective assessment using validated QOL questionnaires. The validated $\mathrm{QOL}$ questionnaires used are NOSE scale and HRQL in rhino surgery.

\section{Materials and Methods}

\section{Study Design and Patient Selection}

A prospective single blinded interventional study to evaluate the functional outcome of septorhinoplasty objectively and subjectively. Eligible participants were patients who presented to Otorhinolaryngology Clinic, Universiti Kebangsaan Malaysia Medical Centre (UKMMC) for evaluation of nasal obstruction and external nasal deformities who fulfilled the inclusion and exclusion criteria from 1st August 2010 to 30th November 2012. The inclusion criteria consisted of patients 18 years and above with the following signs and symptoms of external nasal deformities, persistent nasal obstruction, nasal obstruction that failed medical treatment for more than 6 months, nasal obstruction associated with septal deviation and nasal valve collapse. Patients with sinonasal malignancy, chronic rhinosinusitis, septal perforation, craniofacial syndrome, radiation therapy to the head and neck, septorhinoplasty with concurrent sinus surgery, uncontrolled asthma, acute nasal trauma in the past 3 months, adenoid hypertrophy, rhinomanometer readings within normal range and pregnant females were excluded.

\section{Study Methods and Data Collection}

The study proposal was submitted to Research Ethics Committee Universiti Kebangsaan Malaysia (RECUKM). The study was conducted after the getting the approval from RECUKM. All procedures performed are according to the guidelines and accordance to the ethical standards of RECUKM. A total of 3 visits were scheduled for each patient during the study period; preoperative, operative and postoperative visit. During the pre-operative assessment, the patient was explained and informed consent was taken. Patient's demographic data was filled up in a standardized form. History taking and physical examination was performed to identify the cause of nasal obstruction and the indications for septorhinoplasty. Standard pre-operative photographs were taken and patient was given a Nasal Obstruction Symptoms Evaluation (NOSE) scale and
Health-Related Quality of Life Questionnaire in Rhino Surgery and rhinomanometry measurements obtained prior to the surgery. Septorhinoplasty was performed by same surgeon. Patients were given a clinic appointment to the respective surgeon's clinic 1-2 weeks post surgery for nasal toilet and re-evaluation. Patients were given an appointment 3 months after discharge for postoperative rhinomanometry assessment, NOSE scale and HRQOL Questionnaires in Rhino Surgery. All questionnaires and rhinomanometric data were reviewed during data analysis.

\section{Results}

A total of 35 patients participated in this study and underwent pre-operative assessment. During the study period, there were 6 drop outs $(17.1 \%)$. Due to default of follow up, 3 patients $(8.6 \%)$ were unable to performed rhinomanometry due to septal perforation post-septorhinoplasty ( 2 patients, $5.7 \%$ ) and incomplete data in one patient. Therefore, 29 patients were available for analysis. There were 21 males $(72.4 \%)$ and 8 females $(27.6 \%)$. It was commonly performed in Malays $(\mathrm{n}=14$, $48.3 \%)$, and Indians $(\mathrm{n}=13,44.8 \%)$ and are in Chinese ( $\mathrm{n}=2,6.9 \%)$. Age ranged from 18 to 54 years with mean age of $28.72 \pm 11.38$. Majority of patients did not have history of trauma $(n=16,55.17 \%)$.

The most common external nose abnormality was twisted $(\mathrm{n}=12)$, followed by crooked $(\mathrm{n}=8)$ and saddle nose $(n=6)$. Other abnormalities include depressed tip, bulbous tip, alar deformity and siliconoma (Table 1). One patient had two external nasal abnormalities namely crooked nose with saddle nose. Majority of the patients had deviated nasal septum to left $(\mathrm{n}=19,65.5 \%)$, and $34.5 \%$ $(\mathrm{n}=10)$ to the right. All patients had internal valve insufficiency namely severe anterior deviated nasal septum (DNS) $(\mathrm{n}=22,71 \%)$, upper lateral cartilage collapse $(\mathrm{n}=2,12.9 \%)$, internal nasal valve fibrosis/adhesion $(\mathrm{n}=4,12.9 \%)$ and granulation tissue over upper lateral cartilage $(\mathrm{n}=1,3.2 \%)$. Only $4(13.8 \%)$ patients had external valve insufficiency.

The mean total score of NOSE before the surgery was $3.54 \pm 0.65$. (moderate to fairly bad problem). There was significant improvement of the total NOSE score to $2.06 \pm 0.85$ (very mild to moderate problem) post septorhinoplasty (Fig. 1). Majority of patients had severe problem of nasal obstruction and nasal congestion preoperatively. There were significant improvements in all parameters of NOSE scale after the septorhinoplasty.

HRQOL scores was used to evaluate the impact of nasal obstruction on patient's daily life pre and post septorhinoplasty. The questionnaires measures whether the septorhinoplasty improves the quality of life. The mean 
Table 1 The types of nasal deformities pre septorhinoplasty

\begin{tabular}{lcc}
\hline & $\begin{array}{l}\text { Number } \\
(\mathrm{n})\end{array}$ & $\begin{array}{l}\text { Percentage } \\
(\%)\end{array}$ \\
\hline External nose deformity & 12 & 38.7 \\
$\quad$ Twisted & 8 & 25.8 \\
Crooked & 6 & 19.4 \\
Saddle & 2 & 6.5 \\
Depressed tip & 1 & 3.2 \\
Bulbous tip & 1 & 3.2 \\
Alar deformity & 1 & 3.2 \\
$\quad$ Siliconoma & 10 & 34.5 \\
Nasal septum deviation & 19 & 65.5 \\
$\quad$ Right & & \\
Left & 22 & 71.0 \\
Internal nasal valve insufficiency & 4 & 12.9 \\
Severe anterior deviated nasal septum & 22.9 \\
Upper lateral cartilage collapse & 4 & 12.2 \\
Internal nasal valve fibrosis/adhesion & 4 & 13.8 \\
Granulation tissue over upper lateral & 1 & \\
cartilage & & \\
External valve insufficiency & 4 & \\
\hline
\end{tabular}

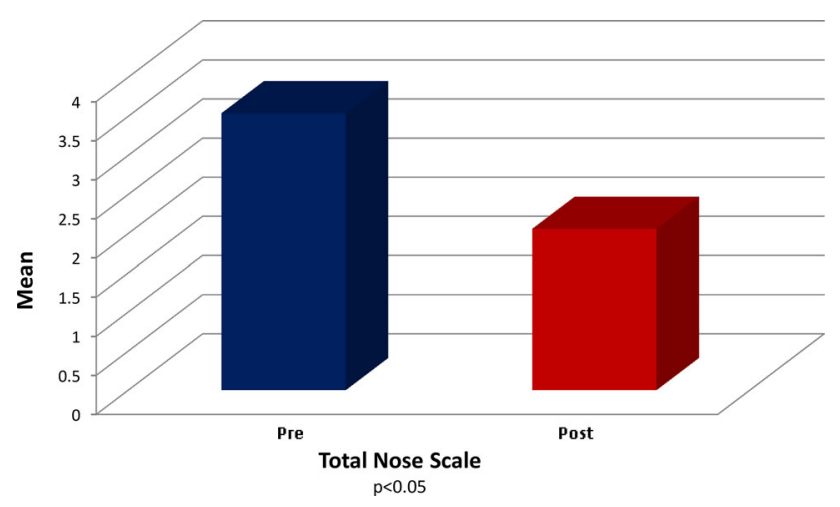

Fig. 1 Comparison of mean score of NOSE pre and post septorhinoplasty

total score of HRQL and all the parameters except headache and activity were normally distributed. The parametric and non-parametric statistical analysis were used for normally and non-normally distribution respectively. From the study, the mean score of total HRQL was $2.38 \pm 0.55$ pre septorhinoplasty and improves significantly post septorhinoplasty (mean: $1.61 \pm 0.45$ ). Furthermore, there were significant improvements in all of the parameters in the HRQL scores including patient's activity, sleep, non nasal symptoms, headache, practical, nasal symptoms and emotion (Fig. 2).

Rhinomanometry measures the resistance during inspiration and expiration. The flow is adversely proportionate to the nasal resistance. Therefore, with reduction in the

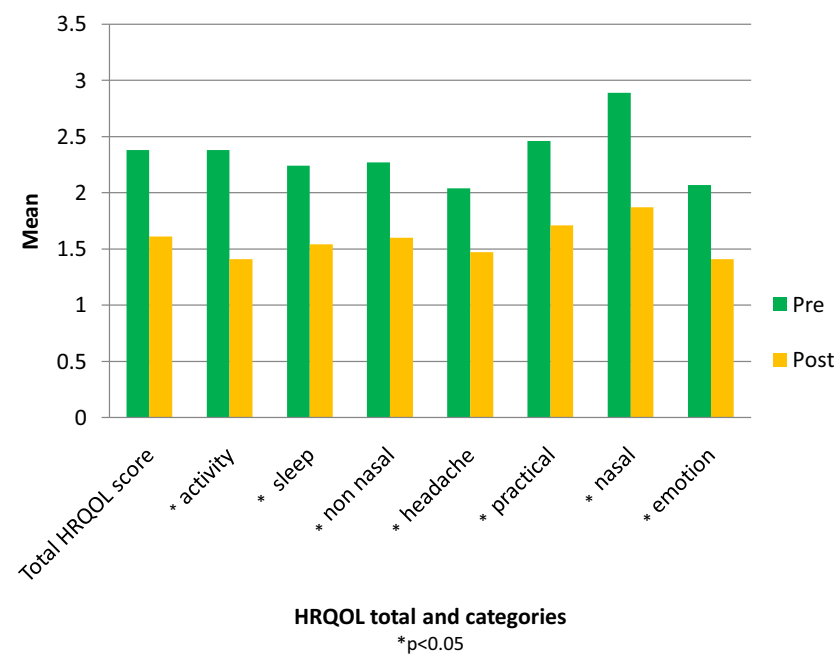

Fig. 2 Comparison of mean score of HRQOL pre and post septorhinoplasty

nasal resistance, the nasal airflow will increase. All of the parameters in the rhinomanometry data except for the inspiratory total were not normally distributed. From the total number of patients, there were improvements in the nasal resistance during inspiration and expiration in both nasal cavities which was not statistically significant (Fig. 3).

In this study, septorhinoplasty improved the NOSE scale, HRQL score and rhinomanometry score (nasal resistance). There is significant correlation in the improvement between the two subjective assessments (NOSE scale and HRQL score). However, there was no significant correlation in the improvement between the subjective (NOSE scale and HRQL score) with objective (rhinomanometry score) assessment (Table 2).

\section{Discussion}

Malaysia is unique with a multiracial and multiethnicity population where the nasal anatomic characteristic varies greatly amongst the Malay, Chinese and Indians. In this study, majority of the patients were Malays followed by Indian and Chinese which varies from our previous series on septorhinoplasty where majority of the patients were of Indian origin [7]. As compared to Malay and Chinese (oriental nose), Indians tend to possess the nasal geometry of the Caucasians due to their ancestral roots. Caucasian noses are more greatly projected at tip and nasion as compared to Oriental noses [8]. The Oriental noses have thick skin, flat dorsum, widened ala base and the nose appears relatively small and flat with bulbous tip [9].

Twisted nose was the most common external nasal deformity in this study and this is similar to previous series 


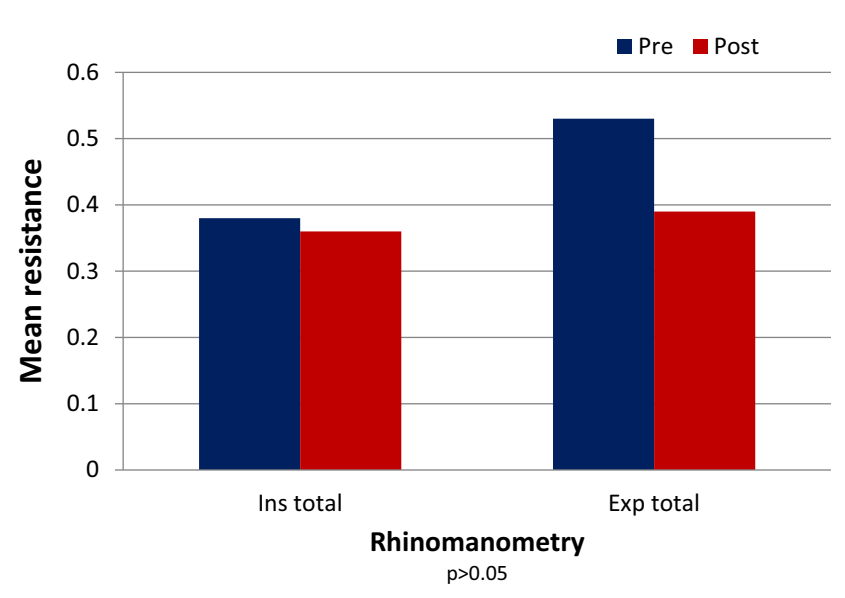

Fig. 3 Mean rhinomanometry comparison data pre and post septorhinoplasty

[7]. The deviated nasal septum considered in this study was the anterior deviation which anatomically is in close relation to the internal nasal valve. All of our patients in this study had anterior deviated nasal septum and few posterior deviations. Nasal valve area constitutes the narrowest part of the nose and also poses the greatest resistance to nasal airflow. The term internal nasal valve insufficiency is used for cases in which reduction of the nasal valve area is caused by a narrowing of the angle between the nasal septum and the upper lateral cartilage [10]. In this study, all of our patients that underwent septorhinoplasty had nasal valve insufficiency.

The nasal valve collapse and deviated nasal septum are part of the cause of nasal obstruction and nasal airway resistance. Patients with nasal valve collapse demonstrated greater perception of nasal obstruction than those with septal deviation alone [11]. Deviated nasal septum and nasal valve collapse are the fixed cause of nasal obstruction; therefore, the mainstay of treatment is surgical correction. In this study, septorhinoplasty with turbinoplasty was performed to correct the deformity and improve nasal obstruction.
We assessed the outcome of septorhinoplasty using rhinomanometry as the objective tool. The Standardization Committee on Objective Assessment of Nasal Airway decided that active anterior rhinomanometry should be the method of choice for measurement of nasal ventilation [12]. To our knowledge, there is no data available concerning rhinomanometry value indicated for septorhinoplasty. The total nasal airway resistance in normal subjects ranged from 0.12 to $0.52 \mathrm{~Pa} / \mathrm{cm}^{3} / \mathrm{s}$ at 150 Pascal pressure point [13]. In this study, the mean nasal resistance were 0.38 and $0.36 \mathrm{~Pa} / \mathrm{cm}^{3} / \mathrm{s}$ pre and post septorhinoplasty respectively at 150 Pascal pressure point. There was improvement in nasal resistance in general post septorhinoplasty but was not statistically significant. We postulated that the data was not statistically significant due to small sample size and associated with reliability and reproducibility issues of rhinomanometry namely the way of application of the nozzle, nasal adaptor and/or face mask, whereas artifacts arising from distortion of the compliant ala segment and functional nasal valve might cause misleading and unreliable data [14]. Other possible reason of inaccuracy of rhinomanometry data are loose face mask, incomplete closure of mouth, stabilization of nasal soft tissue by face masks similar to Cottle-maneuver, improper or lack of calibration method, ventilation technique and calculation force of combined nasal resistance in anterior technique $[14,15]$. Therefore, in this study the standardized technique of ventilation, proper sealed mask and nasal applicator and frequent calibration of the machine were performed to ensure reliable or near reliable data. We measured each nostril for three times and took the average of the measurement to avoid the influence of the nasal cycle.

NOSE scale was used in this study pre and post septorhinoplasty to subjectively evaluate the symptoms of nasal obstruction. It is a validated disease specific quality of life to evaluate the outcome of functional rhinoplasty and septoplasty. In this study, septorhinoplasty that

Table 2 Correlation between improvement of nasal obstruction symptoms and nasal resistance

\begin{tabular}{llll}
\hline & Mean improve NOSE & Mean improve HRQOL & Mean improve rhinomanometry \\
\hline $\begin{array}{l}\text { Mean improve NOSE } \\
\text { Pearson correlation }\end{array}$ & 1 & $0.684^{* *}$ & -0.216 \\
$\quad$ Sig (2-tailed) & & 0.00 & 0.216 \\
Mean improve HRQOL & $0.684^{* *}$ & 1 & -0.107 \\
Pearson correlation & 0.00 & & 0.579 \\
Sig (2-tailed) & & -0.107 & 1 \\
Mean improve insp total & -0.216 & 0.579 & \\
Pearson correlation & 0.216 & & \\
Sig (2-tailed) & & & \\
\hline
\end{tabular}

** $p<0.05$ 
addressed the nasal valve and septum improves the NOSE scale significantly. Patients experience significant improvement of the nasal symptoms 3 months post septorhinoplasty. One study showed significant improvement in NOSE scale score post functional rhinoplasty for internal valve insufficiency [1]. A multi-institutional outcome study revealed that surgical correction of nasal valve dysfunction significantly improves disease specific quality of life based on NOSE scale 3 and 6 months post surgery [16]. However, these studies only address the nasal valve area. The NOSE scale score was also used by Stewart et al. [17] to evaluate the nasal obstruction symptoms pre and post septoplasty and showed significant improvement of nasal obstruction post septoplasty. Therefore, in this study septorhinoplasty was performed to correct the nasal valve and septum simultaneously.

The HRQL in rhino surgery used in this study was used to assess the impact of nasal obstruction to the general quality of life including sleep, non-nasal symptoms, headache, household and outdoor activity, practical and psychological aspect. In our study, septorhinoplasty significantly improves the total score of HRQL and all the parameters within the questionnaires. There are many other questionnaires available for general quality of life in relation to nasal obstruction. Kramer et al. [18] used the HRQL in Rhino Surgery to assess the general quality of life post surgery related to nose including septorhinoplasty. The psychosocial effect of septorhinoplasty must not be underestimated. We found out that there were significant improvement in patient's activity and emotion after septorhinoplasty. However, we did not use specific questionnaires such as Derriford Appearance Scale (DAS-59) for psychosocial assessment which was utilized by Won et al. [19]. Their study found improvement of scores related to general self-consciousness, negative self-concept, and physical stress post septorhinoplasty. If comparison is made between improvement of functional and cosmetic outcome of septorhinoplasty, it revealed that the functional improvement was more superior than cosmetic improvement [20].

There have been several studies which correlates subjective and objective assessment in patients with nasal obstruction. McCaffrey and Kem [21] have reported that there is good correlation between nasal obstruction symptoms and nasal resistance in 1000 patients and that nasal resistance may quantify the nasal obstruction symptoms. However it was reported that there is no correlation between nasal obstruction symptoms and total nasal resistance [22] which was similar to our findings. One of the postulation was that specific nasal sensory nerve endings, probably the major palatine nerve, might be responsible for the subjective perception of nasal patency and can be entirely independent of any objective measurable change in nasal resistance [23]. Jones et al. [24] performed 500 measurements in 250 subjects and concluded that nasal resistance to airflow and nasal sensation of airflow are two separate, indirect modalities.

\section{Conclusion}

In conclusion, septorhinoplasty improves the nasal airflow and quality of life of patients with nasal obstruction. Therefore, septorhinoplasty is the treatment of choice in patients with fixed causes of nasal obstruction due to septal deviation and nasal valve insufficiency. We recommend for bigger sample size and longer duration of study to see the long term outcome of septorhinoplasty. Acoustic rhinometry should be used in the future to measure the nasal dimension post septorhinoplasty. Further study is recommended to compare the outcome of septoplasty with septorhinoplasty or rhinoplasty with septorhinoplasty for patients presenting with nasal obstruction.

Acknowledgments This study was funded by Medical Research and Innovation Secretariat, UKM Medical Centre. Grant Number: FF-101-2011.

\section{Compliance with Ethical Standard}

Conflict of interest Dr. Farah Dayana Zahedi, Associate Professor Dr. Salina Husain and Professor Dato' Dr. Balwant Singh Gendeh declare that they have no conflict of interest.

Ethical approval All procedures performed in this study were in accordance with the ethical standards of Research Ethic Committee Universiti Kebangsaan Malaysia (RECUKM) and with the 1964 Helsinki declaration and its later amendments or comparable ethical standards. Formal consent obtained from each of the individuals in this study.

Informed consent Informed consent together with formal consent were obtained from all individual participants for whom indentifying information is included in this article.

\section{References}

1. Most SP (2006) Analysis of outcomes after functional rhinoplasty using a disease-specific quality-of-life instrument. Arch Facial Plast Surg 8(5):306-309

2. Toriumi DM (1996) The relative importance of septal and nasal valvular surgery in correcting airway obstruction in primary and secondary rhinoplasty. Plast Reconstr Surg 98(1):55-58

3. Rhee JS, Arganbright JM, MacMullin BT, Hannley M (2008) Evidence supporting functional rhinoplasty or nasal valve repair: a 25-year systemic review. Otolaryngol Head Neck Surg 139:10-20

4. Constantian MB, Clardy RB (1996) The relative importance of septal and nasal valvular surgery in correcting airway obstruction in primary and secondary rhinoplasty. Plast Reconstr Surg 98(1):38-54 
5. Ballert JA, Park SS (2006) Functional rhinoplasty: treatment of the dysfunctional nasal sidewall. Facial Plast Surg 22(1):49-54

6. Schwentner I, Dejakum K, Schmutzhard J, Deibl M, Sprinzi GM (2006) Does nasal septal surgery improve quality of life? Acta Oto-Laryngol 126(7):752-757

7. Gendeh BS, Tan VE (2007) Open septorhinoplasty: operative technique and grafts. Med J Malaysia 62(1):13-18

8. Leong SCL, White PS (2004) A comparison of aesthetic proportions between the Oriental and Caucasion nose. Clin Otolaryngol 29(6):672-676

9. Farrior RT, Farrior EH, Cook R (2005) Special rhinoplasty techniques. In: Cummings CW, Flint PW, Harker LA et al (eds) Cummings otolaryngology, head and neck surgery, 4th edn. Elsevier Mosby, Philadelphia, pp 1078-1114

10. Andre RF, Paun SH, Vuyk HD (2004) Endonasal spreader graft placement as treatment for internal nasal valve insufficiency: no need to divide the upper lateral cartilage from the septum. Arch Facial Plast Surg 6:36-40

11. Rhee JS, Book DT, Burzynski M, Smith TL (2003) Quality of life in nasal airway obstruction. Laryngoscope 113(7):1118-1122

12. Clement PA (1984) Committee report on standardization of rhinomanometry. Rhinology 22:151-155

13. Suzina AH, Hamzah M, Samsudin AR (2003) Active anterior rhinomanometer analysis in normal adult Malays. J Laryngol Otol 117:605-608

14. Hirschberg A (2002) Rhinomanometry: an update. ORL 64:263-267

15. Bermuller C, Kirsche H, Rettinger G, Riechelmann H (2008) Diagnostic accuracy of peak nasal inspiratory flow and rhinomanometry in functional rhinosurgery. Laryngoscope 118(4): $605-610$
16. Rhee JS, Poetker DM, Smith TL, Bustillo A, Burzynski M, Dabis RE (2005) Nasal valve surgery improves disease-specific quality of life. Laryngoscope 115(3):437-440

17. Stewart MG, Witsell DL, Smith TL, Weaver EM, Yueh B, Hannley MT (2004) Development and validation of Nasal Obstruction Symptom Evaluation (NOSE) Scale. Otolaryngol Head Neck Surg 130(2):157-163

18. Kramer MF, Rasp G, Kastenbauer E (2003) Health-related quality of life in rhino surgery. Am J Otolaryngol 24(2):97-105

19. Won TB, Park KT, Moon SJ, Moon IJ, Wee JH, Moss T, Jin HR (2013) The effect of septorhinoplasty on quality of life and nasal function in Asians. Ann Plast Surg 71(1):40-44. doi: 10.1097/SAP.0b013e3182414641

20. Gendeh BS, Mallina S (2008) Graft selection in rhinoplasty: indication and limitations. Med J Malaysia 63(1):35-38

21. McCaffrey TV, Kern EB (1979) Clinical evaluation of nasal obstruction, a study of 1000 patients. Arch Otolaryngol 105(9):542-545

22. Sipila J, Suonpaa J, Silvoniemi P, Laippala P (1995) Correlation between subjective sensation of nasal patency and rhinomanometry in both unilateral and total nasal assessment. ORL 57(5):260-263

23. Andre RF, Vuyk HD, Ahmed A, Graamans K, Nolst Trenite GJ (2009) Correlation between subjective and objective evaluation of the nasal airway. A systematic review of the highest level of evidence. Clin Otolaryngol 34:518-525

24. Jones AS, Wight RG, Crosher R, Durham LH (1989) Nasal sensation of airflow following blockade of the nasal trigeminal afferents. Clin Otolaryngol 14(4):285-289 